\title{
Remarks about a construction method for D-optimal chemical balance weighing designs
}

\author{
Małgorzata Graczyk, Malwina Janiszewska
}

\begin{abstract}
Department of Mathematical and Statistical Methods, Poznań University of Life Sciences, Wojska Polskiego 28, 60-638 Poznań, Poland, e-mails: malgorzata.graczyk@up.poznan.pl, malwina.janiszewska@up.poznan.pl
\end{abstract}

\section{SUMMARY}

\begin{abstract}
This paper presents some constructions of regular D-optimal weighing designs based on the incidence matrices of a balanced incomplete block design, balanced bipartite weighing design and ternary balanced block design. We determine optimality conditions and relations between the parameters of the design, and give an example.
\end{abstract}

Keywords and phrases: balanced bipartite weighing design, balanced incomplete block design, chemical balance weighing design, D-optimality, ternary balanced block design

\section{Introduction}

We consider here the linear model

$$
y=X w+e,
$$

where $\mathbf{y}$ is an $n \times 1$ random vector of observations, $\mathbf{X} \in \mathbf{\Phi}_{n \times p}(-1,0,1)$ (the class of $n \times p$ matrices $), \mathbf{X}=\left(x_{i j}\right)$ consists of known elements, where

$$
x_{i j}=\left\{\begin{array}{cc}
-1 & \begin{array}{r}
\text { if the } j^{\text {th }} \text { object in the } i^{\text {th }} \text { weighing operation } \\
\text { is placed in the left pan }
\end{array} \\
0 & \begin{array}{r}
\text { if the } j^{\text {th }} \text { object in the } i^{\text {th }} \text { weighing operation } \\
\text { is not placed in either pan }
\end{array} \\
1 & \begin{array}{r}
\text { if the } j^{\text {th }} \text { object in the } i^{\text {th }} \text { weighing operation } \\
\text { is placed in the right pan }
\end{array}
\end{array}\right.
$$

in the case of a chemical balance, $\mathbf{w}$ is a $p \times 1$ vector of unknown measurements of objects, and $\mathbf{e}$ is an $n \times 1$ random vector of errors. We assume that $\mathrm{E}(\mathbf{e})=\mathbf{0}_{n}$ 
and $\operatorname{Var}(\mathbf{e})=\sigma^{2} \mathbf{I}_{n}$, where $\mathbf{0}_{n}$ is the $n \times 1$ vector with zero elements everywhere, and $\mathbf{I}_{n}$ denotes the identity matrix of rank $n$. Such a form of the matrix $\operatorname{Var}(\mathbf{e})$ indicates that the errors are uncorrelated and have the same variance.

For the estimation of $\mathbf{w}$ we use the least squares method. As a result the normal equations are of the form $\mathbf{X}^{\prime} \mathbf{X} \widehat{\mathbf{w}}=\mathbf{X}^{\prime} \mathbf{y}$. A chemical balance weighing design is singular or nonsingular, depending on whether the matrix $\mathbf{X}^{\prime} \mathbf{X}$ is singular or not singular. If $\mathbf{X}$ is of full column rank, the least squares estimator of $\mathbf{w}$ is equal to $\widehat{\mathbf{w}}=(\mathbf{M})^{-\mathbf{1}} \mathbf{X}^{\prime} \mathbf{y}$ and the covariance matrix of $\widehat{\mathbf{w}}$ is given by $\operatorname{Var}(\widehat{\mathbf{w}})=\sigma^{2}(\mathbf{M})^{-1}$, where $\mathbf{M}=\mathbf{X}^{\prime} \mathbf{X}$ is called the information matrix for the $\operatorname{design} \mathbf{X}$.

In the literature, basic problems of weighing designs are discussed. Jacroux et al. (1983) and Sathe and Shenoy (1990) introduced different optimality criteria, and new methods of construction for design matrices satisfying optimality conditions are presented by Ceranka and Graczyk (2010, 2012). The relations between a chemical balance weighing design and the factorial design are considered by Cheng et al. (2004).

In this paper, we consider D-optimal designs. The design $\mathbf{X}_{D}$ is called D-optimal in the given class $\boldsymbol{\Phi}_{n \times p}(-1,0,1)$ if

$$
\operatorname{det}\left(\boldsymbol{X}_{D}^{\prime} \boldsymbol{X}_{D}\right)=\max \left(\operatorname{det}(\boldsymbol{M}): \boldsymbol{X} \in \boldsymbol{\Phi}_{n \times p}(-1,0,1)\right) .
$$

If $\operatorname{det}(\mathbf{M})$ attains the upper bound, the design is called regular D-optimal. In other cases, such a design is called D-optimal. Every regular D-optimal design is D-optimal, but the inverse may not hold. For more theory, we refer the reader to the papers of Katulska and Smaga (2013a, b) and Ceranka and Graczyk (2016).

Theorem 1.1. (Ceranka and Graczyk, 2017) Any chemical balance weighing design $\mathbf{X} \in \mathbf{\Phi}_{n \times p}(-1,0,1)$ with the covariance matrix of errors $\sigma^{2} \mathbf{I}_{n}$ is regular D-optimal if and only if $\mathbf{X}^{\prime} \mathbf{X}=m \mathbf{I}_{p}$, where $m$ is the maximal number of elements different from zero in the $j^{\text {th }}$ column, where $j=1, \ldots, p$.

The aim of this paper is to investigate a construction method for D-optimal chemical balance weighing designs. We will use the incidence matrices of a balanced incomplete block design, balanced bipartite weighing design and 
ternary balanced block design. The basic properties of these designs are presented in section 2. Section 3 contains construction methods for the design matrix of the chemical balance weighing design based on the aforementioned block matrices. Finally, some case studies are given.

\section{Balanced block design}

In this section, we recall the definition and the properties of the balanced incomplete block design given by Raghavaro (1971), those of the balanced bipartite weighing design given by Huang (1976), and those of the ternary balanced block design given by Billington (1984).

In a balanced incomplete block design (BIBD) with parameters $v, b, r, k$, $\lambda$, there is an arrangement of $v$ treatments into $b$ blocks, each of size $k$, in such a way that each treatment occurs at most once in each block and occurs in exactly $r$ blocks, and every pair of treatments occurs together in exactly $\lambda$ blocks. Let $\mathbf{N}$ be the incidence matrix of a balanced incomplete block design. The parameters are related by the following identities:

$$
v r=b k, \lambda(v-1)=r(k-1), \boldsymbol{N N}^{\prime}=(r-\lambda) \boldsymbol{I}_{v}+\lambda \mathbf{1}_{v} \mathbf{1}_{v}^{\prime},
$$

where $\mathbf{1}_{v}$ is the $v \times 1$ vector of ones.

In a balanced bipartite weighing design (BBWD) with parameters $v, b, r$, $k_{1}, k_{2}, \lambda_{1}, \lambda_{2}$, there is an arrangement of $v$ treatments into $b$ blocks such that each block containing $k$ distinct treatments is divided into 2 subblocks containing $k_{1}$ and $k_{2}$ treatments respectively. Each treatment appears in $r$ blocks. Every pair of treatments from different subblocks appears together in $\lambda_{1}$ blocks, and every pair of treatments from the same subblock appears together in $\lambda_{2}$ blocks. Let $\mathbf{N}^{*}$ be the incidence matrix of such a design. The parameters are not independent; they are related by the following equalities: $v r=b k$,

$$
\begin{aligned}
& b=\frac{\lambda_{1} v(v-1)}{2 k_{1} k_{2}}, \lambda_{2}=\frac{\lambda_{1}\left[k_{1}\left(k_{1}-1\right)+k_{2}\left(k_{2}-1\right)\right]}{2 k_{1} k_{2}}, r=\frac{\lambda_{1} k(v-1)}{2 k_{1} k_{2}}, \\
& \boldsymbol{N}^{*} \boldsymbol{N}^{*^{\prime}}=\left(r-\lambda_{1}-\lambda_{2}\right) \boldsymbol{I}_{v}+\left(\lambda_{1}+\lambda_{2}\right) \mathbf{1}_{v} \mathbf{1}_{v}^{\prime} .
\end{aligned}
$$


In a ternary balanced block design (TBBD) with parameters $v, b, r, k, \lambda$, $\rho_{1}, \rho_{2}$ there is an arrangement of $v$ treatments in $b$ blocks each of size $k$ in such a way that each treatment appears 0,1 or 2 times in a given block, and is repeated $r$ times. Each distinct pair of treatments occurs $\lambda$ times. Each element appears once in $\rho_{1}$ block and twice in $\rho_{2}$ blocks, where $\rho_{1}$ and $\rho_{2}$ are known constants for the design. Let $\mathbf{N}$ be the incidence matrix of a ternary balanced block design. The following relations are satisfied:

$$
\begin{aligned}
& v r=b k, r=\rho_{1}+2 \rho_{2}, \\
& \lambda(v-1)=\rho_{1}(k-1)+2 \rho_{2}(k-2)=r(k-1)-2 \rho_{2}, \\
& \boldsymbol{N} \boldsymbol{N}^{\prime}=\left(\rho_{1}+4 \rho_{2}-\lambda\right) \boldsymbol{I}_{v}+\lambda \mathbf{1}_{v} \mathbf{1}_{v}^{\prime}=\left(r+2 \rho_{2}-\lambda\right) \boldsymbol{I}_{v}+\lambda \mathbf{1}_{v} \mathbf{1}_{v}^{\prime} .
\end{aligned}
$$

\section{Construction}

Determining the plan of the experiment is equivalent to establishing the matrix $\mathbf{X}$, where $\mathbf{X}$ is called the design matrix. To broaden the number of classes in which we establish a regular D-optimal chemical balance weighing design, we create the matrix $\mathbf{X}$. It is not possible to give a design matrix satisfying the required optimality criteria for any number of objects and any number of measurements. Therefore, the aim of this work is to determine a D-optimal chemical balance weighing design in a given class $\boldsymbol{\Phi}_{n \times p}(-1,0,1)$, i.e. to give the design matrix for a fixed number of objects $p$ and measures $n$.

Let $\mathbf{N}_{1}$ be the incidence matrix of a BIBD with the parameters $v, b_{1}, r_{1}, k_{1}$, $\lambda_{1}$, and let $\mathbf{N}_{2}^{*}$ be the incidence matrix of a BBWD with the parameters $v, b_{2}, r_{2}$, $k_{12}, k_{22}, \lambda_{12}, \lambda_{22}$. Let us define $\mathbf{N}_{2}$ as obtained from $\mathbf{N}_{2}^{*}$ by replacing $k_{12}$ elements equal to +1 in each column which correspond to the elements belonging to the first subblock by -1 . Consequently, each column of $\mathbf{N}_{2}$ will contain $k_{12}$ elements equal to $-1, k_{22}$ elements equal to 1 and $v-k_{12}-k_{12}$ elements equal to 0. Furthermore, let $\mathbf{N}_{3}$ be the incidence matrix of a TBBD with parameters $v, b_{3}, r_{3}, k_{3}, \lambda_{3}, \rho_{13}, \rho_{23}$. Then the design matrix $\mathbf{X} \in \mathbf{\Phi}_{n \times p}(-1,0,1)$ has the form 


$$
\boldsymbol{X}=\left[\begin{array}{c}
2 \boldsymbol{N}_{1}^{\prime}-\mathbf{1}_{b_{1}} \mathbf{1}_{v}^{\prime} \\
\boldsymbol{N}_{2}^{\prime} \\
\boldsymbol{N}_{3}^{\prime}-\mathbf{1}_{b_{3}} \mathbf{1}_{v}^{\prime}
\end{array}\right]
$$

Each of the $p=v$ objects is weighed $m=b_{1}+r_{2}+b_{3}-\rho_{13}$ times in $n=b_{1}+b_{2}+b_{3}$ measuring operations.

Lemma 3.1. Any chemical balanced weighing design $\mathbf{X} \in \boldsymbol{\Phi}_{n \times p}(-1,0,1)$ as given in (1) is nonsingular if and only if $2 k_{1} \neq v$ or $v \neq k_{3}$ or $2 k_{1} \neq k_{3}$ or $k_{12} \neq k_{22}$.

Proof. Consider the design matrix $\mathbf{X} \in \mathbf{\Phi}_{n \times p}(-1,0,1)$ in the form (1). We shall prove the Lemma if we give the determinant of the information matrix for the design. We have

$$
\begin{aligned}
& \boldsymbol{X}^{\prime} \boldsymbol{X}=4 \boldsymbol{N}_{1}^{\prime} \boldsymbol{N}_{1}-2 \boldsymbol{N}_{1} \mathbf{1}_{b_{1}} \mathbf{1}_{v}^{\prime}-2 \mathbf{1}_{v} \mathbf{1}_{b_{1}}^{\prime} \boldsymbol{N}_{1}^{\prime}+\mathbf{1}_{v} \mathbf{1}_{b_{1}}^{\prime} \mathbf{1}_{b_{1}} \mathbf{1}_{v}^{\prime}+\boldsymbol{N}_{2} \boldsymbol{N}_{2}^{\prime}+ \\
& \boldsymbol{N}_{3} \boldsymbol{N}_{3}^{\prime}-\boldsymbol{N}_{3} \mathbf{1}_{b_{3}} \mathbf{1}_{v}^{\prime}-\mathbf{1}_{v} \mathbf{1}_{b_{3}}^{\prime} \boldsymbol{N}_{3}^{\prime}+\mathbf{1}_{v} \mathbf{1}_{b_{3}}^{\prime} \mathbf{1}_{b_{3}} \mathbf{1}_{v}^{\prime} \\
& =4\left(r_{1}-\lambda_{1}\right) \boldsymbol{I}_{v}+4 \lambda_{1} \mathbf{1}_{v} \mathbf{1}_{v}^{\prime}-2 r_{1} \mathbf{1}_{v} \mathbf{1}_{v}^{\prime}-2 r_{1} \mathbf{1}_{v} \mathbf{1}_{v}^{\prime}+b_{1} \mathbf{1}_{v} \mathbf{1}_{v}^{\prime}+ \\
& \left(r_{2}-\lambda_{22}+\lambda_{12}\right) \boldsymbol{I}_{v}+\left(\lambda_{22}-\lambda_{12}\right) \mathbf{1}_{v} \mathbf{1}_{v}^{\prime}+\left(r_{3}+2 \rho_{23}-\lambda_{3}\right) \boldsymbol{I}_{v}+ \\
& \lambda_{3} \mathbf{1}_{v} \mathbf{1}_{v}^{\prime}-r_{3} \mathbf{1}_{v} \mathbf{1}_{v}^{\prime}-r_{3} \mathbf{1}_{v} \mathbf{1}_{v}^{\prime}+b_{3} \mathbf{1}_{v} \mathbf{1}_{v}^{\prime} \\
& =\left(4\left(r_{1}-\lambda_{1}\right)+r_{2}-\lambda_{22}+\lambda_{12}+r_{3}+2 \rho_{23}-\lambda_{3}\right) \boldsymbol{I}_{v}+\left(b_{1}-\right. \\
& \left.4\left(r_{1}-\lambda_{1}\right)+\lambda_{22}-\lambda_{12}+b_{3}-2 r_{3}+\lambda_{3}\right) \mathbf{1}_{v} \mathbf{1}_{v}^{\prime}
\end{aligned}
$$

and

$$
\begin{aligned}
& \operatorname{det}\left(\boldsymbol{X}^{\prime} \boldsymbol{X}\right)=\left(4\left(r_{1}-\lambda_{1}\right)+r_{2}-\lambda_{22}+\lambda_{12}+r_{3}+2 \rho_{23}-\lambda_{3}\right)^{v-1} \\
& \left(\frac{r_{1}}{k_{1}}\left(v-2 k_{1}\right)^{2}+\frac{(v-1) \lambda_{12}}{2 k_{12} k_{22}}\left(k_{12}-k_{22}\right)^{2}+\frac{r_{3}}{k_{3}}\left(v-k_{3}\right)^{2}\right)
\end{aligned}
$$

Because $4\left(r_{1}-\lambda_{1}\right)+r_{2}-\lambda_{22}+\lambda_{12}+r_{3}+2 \rho_{23}-\lambda_{3}>0$, the determinant is equal to 0 if and only if $v=2 k_{1}=k_{3}$ and $k_{12}=k_{22}$.

Theorem 3.1. Any nonsingular chemical balance weighing design $\mathbf{X} \in \boldsymbol{\Phi}_{n \times p}(-1,0,1)$ as given in (1) is regular D-optimal if and only if

$$
b_{1}-4\left(r_{1}-\lambda_{1}\right)+\lambda_{22}-\lambda_{12}+b_{3}-2 r_{3}+\lambda_{3}=0 .
$$


Proof. Applying Theorem 1.1 and Lemma 3.1 together, we observe that the information matrix of the design is proportional to the identity matrix. In particular, the equality (2) holds when $b_{1}=4\left(r_{1}-\lambda_{1}\right), \lambda_{22}=\lambda_{12}$ and $b_{3}=2 r_{3}-\lambda_{3}$, is true when any combination of these parameters in total gives zero. Based on the series of parameters given by Ceranka and Graczyk (2004a, b) for the block designs presented in section 2 , we formulate the following corollaries.

Corollary 3.1. Let $v=4$. The existence of the balanced incomplete block design with the parameters

$b_{1}=4 t, r_{1}=3 t, k_{1}=3, \lambda_{1}=2 t, t=1,2, \ldots$ or

$b_{1}=4 t, r_{1}=t, k_{1}=1, \lambda_{1}=0, t=1,2, \ldots$,

and the balanced bipartite weighing design with the parameters $b_{2}=r_{2}=$ $4(2 s-1), k_{12}=1, k_{22}=3, \lambda_{12}=\lambda_{22}=2(2 s-1), s=1,2, \ldots$, and also the ternary balanced block design with the parameters $b_{3}=4 z, r_{3}=5 z, k_{3}=5$, $\lambda_{3}=6 z, \rho_{13}=3 z, \rho_{23}=z, z=1,2, \ldots$, implies the existence of the regular D-optimal chemical balance weighing design $\mathbf{X} \in \boldsymbol{\Phi}_{n \times p}(-1,0,1)$ in (1) with the variance matrix of errors $\sigma^{2} \mathbf{I}_{n}$.

Corollary 3.2. Let $v=5$. If the parameters of the balanced incomplete block design are equal to $b_{1}=10, r_{1}=4, k_{1}=2, \lambda_{1}=1$, and the parameters of the balanced bipartite weighing design are equal to $b_{2}=r_{2}=5, k_{12}=1, k_{22}=4$, $\lambda_{12}=2, \lambda_{22}=3$ and the parameters of the ternary balanced block design are equal to $b_{3}=5 z, r_{3}=3 z, k_{3}=3, \lambda_{3}=z+1, \rho_{13}=z+4, \rho_{23}=z-2$, $z=3,4,5$, then $\mathbf{X} \in \boldsymbol{\Phi}_{n \times p}(-1,0,1)$ in the form (1) with the variance matrix of errors $\sigma^{2} \mathbf{I}_{n}$ is a regular D-optimal chemical balance weighing design.

Corollary 3.3. Let $v=6$. The existence of the balanced incomplete block design with the parameters:

$b_{1}=10, r_{1}=5, k_{1}=3, \lambda_{1}=2$ or

$b_{1}=30, r_{1}=10, k_{1}=\lambda_{1}=2$

and the balanced bipartite weighing design with the parameters 
$b_{2}=15, r_{2}=10, k_{12}=1, k_{22}=\lambda_{12}=\lambda_{22}=3$ or

$b_{2}=30, r_{2}=20, k_{12}=1, k_{22}=3, \lambda_{12}=\lambda_{22}=6$

and also the ternary balanced block design with the parameters

$b_{3}=12, r_{3}=6, k_{3}=3, \lambda_{3}=2, \rho_{13}=4, \rho_{23}=1$ or

$b_{3}=14, r_{3}=7, k_{3}=3, \lambda_{3}=2, \rho_{13}=3, \rho_{23}=2$

implies the existence of the regular D-optimal chemical balance weighing design $\mathbf{X} \in \boldsymbol{\Phi}_{n \times p}(-1,0,1)$ in (1) with the variance matrix of errors $\sigma^{2} \mathbf{I}_{n}$.

Corollary 3.4. Let $v=6$. The existence of the balanced incomplete block design with the parameters:

$b_{1}=10, r_{1}=5, k_{1}=3, \lambda_{1}=2$ or

$b_{1}=30, r_{1}=10, k_{1}=\lambda_{1}=2$

and the balanced bipartite weighing design with the parameters $b_{2}=r_{2}=6$, $k_{12}=1, k_{22}=5, \lambda_{12}=2, \lambda_{22}=4$ and also the ternary balanced block design with the parameters $b_{3}=6 s, r_{3}=4 s, k_{3}=4, \lambda_{3}=\rho_{13}=2 s, \rho_{23}=s$, $s=1,2, \ldots$, implies the existence of the regular D-optimal chemical balance weighing design $\mathbf{X} \in \mathbf{\Phi}_{n \times p}(-1,0,1)$ in (1) with the variance matrix of errors $\sigma^{2} \mathbf{I}_{n}$

Corollary 3.5. Let $v=6$. The existence of the balanced incomplete block design with the parameters $b_{1}=15, r_{1}=5, k_{1}=2, \lambda_{1}=1$ and the balanced bipartite weighing design with the parameters $b_{2}=r_{2}=15, k_{12}=k_{22}=2$, $\lambda_{12}=8, \lambda_{22}=7$ and also the ternary balanced block design with the parameters:

$b_{3}=12, r_{3}=6, k_{3}=3, \lambda_{3}=2, \rho_{13}=1, \rho_{23}=4$ or

$b_{3}=14, r_{3}=7, k_{3}=3, \lambda_{3}=2, \rho_{13}=3, \rho_{23}=2$

implies the existence of the regular D-optimal chemical balance weighing design $\mathbf{X} \in \boldsymbol{\Phi}_{n \times p}(-1,0,1)$ in (1) with the variance matrix of errors $\sigma^{2} \mathbf{I}_{n}$.

Corollary 3.6. Let $v=6$. The existence of the balanced incomplete block design with the parameters $b_{1}=15, r_{1}=5, k_{1}=2, \lambda_{1}=1$ and the balanced bipartite weighing design with the parameters 


$$
\begin{aligned}
& b_{2}=15, r_{2}=10, k_{12}=1, k_{22}=\lambda_{12}=\lambda_{22}=3 \text { or } \\
& b_{2}=30, r_{2}=20, k_{12}=1, k_{22}=3, \lambda_{12}=\lambda_{22}=6 \text { and }
\end{aligned}
$$

also the ternary balanced block design with the parameters $b_{3}=21, r_{3}=14$, $k_{3}=4, \lambda_{3}=8, \rho_{13}=12, \rho_{23}=1$ implies the existence of the regular D-optimal chemical balance weighing design $\mathbf{X} \in \mathbf{\Phi}_{n \times p}(-1,0,1)$ in (1) with the variance matrix of errors $\sigma^{2} \mathbf{I}_{n}$.

Corollary 3.7. Let $v=7$. The existence of the balanced incomplete block design with the parameters $b_{1}=7, r_{1}=k_{1}=3, \lambda_{1}=1$ and the balanced bipartite weighing design with the parameters $b_{2}=r_{2}=21, k_{12}=2, k_{22}=5$, $\lambda_{12}=10, \lambda_{22}=11$, and also the ternary balanced block design with the parameters $b_{3}=7 z, r_{3}=5 z, k_{3}=5, \lambda_{3}=\rho_{13}=3 z, \rho_{23}=z, z=1,2, \ldots$, implies the existence of the regular D-optimal chemical balance weighing design $\mathbf{X} \in \mathbf{\Phi}_{n \times p}(-1,0,1)$ in (1) with the variance matrix of errors $\sigma^{2} \mathbf{I}_{n}$

Corollary 3.8. Let $v=7$ The existence of the balanced incomplete block design with the parameters $b_{1}=21, r_{1}=6, k_{1}=2, \lambda_{1}=1$ and the balanced bipartite weighing design with the parameters

$b_{2}=r_{2}=7, k_{12}=3, k_{22}=\lambda_{12}=4, \lambda_{22}=3$ or

$b_{2}=21, r_{2}=9 s, k_{12}=s, k_{22}=2 s, \lambda_{12}=2 s^{2}, \lambda_{22}=\frac{s(5 s-3)}{2}, s=1,2$

and also the ternary balanced block design with the parameters

$b_{3}=7 z, r_{3}=5 z, \quad k_{3}=5, \lambda_{3}=\rho_{13}=3 z, \rho_{23}=z, z=1,2, \ldots$ or

$b_{3}=14, r_{3}=16, k_{3}=8, \lambda_{3}=18, \rho_{13}=12, \rho_{23}=2$,

implies the existence of the regular D-optimal chemical balance weighing design $\mathbf{X} \in \mathbf{\Phi}_{n \times p}(-1,0,1)$ in (1) with the variance matrix of errors $\sigma^{2} \mathbf{I}_{n}$.

Corollary 3.9. Let $v=7$. The existence of the balanced incomplete block design with the parameters $b_{1}=21, r_{1}=6, k_{1}=2, \lambda_{1}=1$ and the balanced bipartite weighing design with the parameters $b_{2}=7 s, r_{2}=4 s, k_{12}=1$, $k_{22}=3, \lambda_{12}=\lambda_{22}=s, s=1,2, \ldots$, and also the ternary balanced block design with the parameters

$b_{3}=r_{3}=z+6, k_{3}=7, \lambda_{3}=z+5, \rho_{13}=z, \rho_{23}=3, z=1,2, \ldots$ or 
$b_{3}=14, r_{3}=12, k_{3}=6, \lambda_{3}=9, \rho_{13}=6, \rho_{23}=3$ or

$b_{3}=21, r_{3}=15, k_{3}=5, \lambda_{3}=8, \rho_{13}=3, \rho_{23}=6$,

implies the existence of the regular D-optimal chemical balance weighing design $\mathbf{X} \in \mathbf{\Phi}_{n \times p}(-1,0,1)$ in (1) with the variance matrix of errors $\sigma^{2} \mathbf{I}_{n}$.

Corollary 3.10. Let $v=7$. The existence of the balanced incomplete block design with the parameters $b_{1}=21, r_{1}=6, k_{1}=2, \lambda_{1}=1$ and the balanced bipartite weighing design with the parameters $b_{2}=r_{2}=21, k_{12}=2, k_{22}=5$, $\lambda_{12}=10, \lambda_{22}=11$, and also the ternary balanced block design with the parameters $b_{3}=r_{3}=z+12, \quad k_{3}=7, \quad \lambda_{3}=z+10, \rho_{13}=z, \rho_{23}=6, \quad z=1,2, \ldots$ implies the existence of the regular D-optimal chemical balance weighing design $\mathbf{X} \in \boldsymbol{\Phi}_{n \times p}(-1,0,1)$ in (1) with the variance matrix of errors $\sigma^{2} \mathbf{I}_{n}$.

Corollary 3.11. Let $v=7$. The existence of the balanced incomplete block design with the parameters $b_{1}=42, r_{1}=12, k_{1}=\lambda_{1}=2$ and the balanced bipartite weighing design with the parameters

$b_{2}=r_{2}=7, k_{12}=3, k_{22}=\lambda_{12}=4, \lambda_{22}=3$ or $b_{2}=7, r_{2}=6, k_{12}=k_{22}=\lambda_{12}=3, \lambda_{22}=2$ or $b_{2}=21, r_{2}=9 s, k_{12}=s, k_{22}=2 s, \lambda_{12}=2 s^{2}, \lambda_{22}=\frac{s(5 s-3)}{2}, s=1,2$ and also the ternary balanced block design with the parameters $b_{3}=r_{3}=z+6, k_{3}=7, \lambda_{3}=z+5, \rho_{13}=z, \rho_{23}=3, z=1,2, \ldots$ or $b_{3}=14, r_{3}=12, k_{3}=6, \lambda_{3}=9, \rho_{13}=6, \rho_{23}=3$ or $b_{3}=21, r_{3}=15, k_{3}=5, \lambda_{3}=8, \rho_{13}=3, \rho_{23}=6$, implies the existence of the regular D-optimal chemical balance weighing design $\mathbf{X} \in \boldsymbol{\Phi}_{n \times p}(-1,0,1)$ in (1) with the variance matrix of errors $\sigma^{2} \mathbf{I}_{n}$.

Corollary 3.12. Let $v=7$. If the parameters of the balanced incomplete block design are equal to $b_{1}=7, r_{1}=k_{1}=3, \lambda_{1}=1$ and the parameters of the balanced bipartite weighing design are equal to $b_{2}=21, r_{2}=15, k_{12}=1$, $k_{22}=\lambda_{12}=4, \lambda_{22}=6$, and also the parameters of the ternary balanced block design are equal to $b_{3}=r_{3}=z+6, k_{3}=7, \lambda_{3}=z+5, \rho_{13}=z, \rho_{23}=3, z=1,2, \ldots$ or 
$b_{3}=14, r_{3}=12, k_{3}=6, \lambda_{3}=9, \rho_{13}=6, \rho_{23}=3$ or

$b_{3}=21, r_{3}=15, k_{3}=5, \lambda_{3}=8, \rho_{13}=3, \rho_{23}=6$,

then $\mathbf{X} \in \mathbf{\Phi}_{n \times p}(-1,0,1)$ in the form (1) with the variance matrix of errors $\sigma^{2} \mathbf{I}_{n}$ is a regular $\mathrm{D}$-optimal chemical balance weighing design.

Corollary 3.13. Let $v=7$. If the parameters of the balanced incomplete block design are equal to $b_{1}=7, r_{1}=k_{1}=3, \lambda_{1}=1$ and the parameters of the balanced bipartite weighing design are equal to

$b_{2}=r_{2}=7, k_{12}=3, k_{22}=\lambda_{12}=4, \lambda_{22}=3$ or

$b_{2}=7, r_{2}=6, k_{12}=k_{22}=\lambda_{12}=3, \lambda_{22}=2$ or

$b_{2}=21, r_{2}=9 s, k_{12}=s, k_{22}=2 s, \lambda_{12}=2 s^{2}, \lambda_{22}=\frac{s(5 s-3)}{2}, s=1,2$,

and also the ternary balanced block design with the parameters $b_{3}=21, r_{3}=12$, $k_{3}=4, \lambda_{3}=5, \rho_{13}=6, \rho_{23}=3$, then $\mathbf{X} \in \boldsymbol{\Phi}_{n \times p}(-1,0,1)$ in the form (1) with the variance matrix of errors $\sigma^{2} \mathbf{I}_{n}$ is a regular D-optimal chemical balance weighing design.

Corollary 3.14. Let $v=8$. If the parameters of the balanced incomplete block design are equal to $b_{1}=14, r_{1}=7, k_{1}=4, \lambda_{1}=3$ and the parameters of the balanced bipartite weighing design are equal to $b_{2}=56, r_{2}=49, k_{12}=2$, $k_{22}=5, \lambda_{12}=20, \lambda_{22}=22$ and also the ternary balanced block design with the parameters $b_{3}=8 s, r_{3}=6 s, k_{3}=6, \lambda_{3}=\rho_{13}=4 s, \rho_{23}=s, s=1,2, \ldots$, then $\mathbf{X} \in \boldsymbol{\Phi}_{n \times p}(-1,0,1)$ in the form (1) with the variance matrix of errors $\sigma^{2} \mathbf{I}_{n}$ is a regular D-optimal chemical balance weighing design.

Corollary 3.15. Let $v=8$. If the parameters of the balanced incomplete block design are equal to $b_{1}=14, r_{1}=7, k_{1}=4, \lambda_{1}=3$ and the parameters of the balanced bipartite weighing design are equal to $b_{2}=28, r_{2}=14, k_{12}=1$, $k_{22}=\lambda_{12}=\lambda_{22}=3$ and also the ternary balanced block design has the parameters $b_{3}=2-4, r_{3}=15, k_{3}=5, \lambda_{3}=8, \rho_{13}=11, \rho_{23}=2$, then $\mathbf{X} \in \boldsymbol{\Phi}_{n \times p}(-1,0,1)$ in the form (1) with the variance matrix of errors $\sigma^{2} \mathbf{I}_{n}$ is a regular D-optimal chemical balance weighing design. 
Corollary 3.16. Let $v=9$. If the parameters of the balanced incomplete block design are equal to $b_{1}=12 t, r_{1}=4 t, k_{1}=3, \lambda_{1}=t, t=1,2, \ldots$, and the parameters of the balanced bipartite weighing design are equal to

$b_{2}=36, r_{2}=16, k_{12}=1, k_{22}=\lambda_{12}=\lambda_{22}=3$ or $b_{2}=36, r_{2}=36, k_{12}=3, k_{22}=6, \lambda_{12}=\lambda_{22}=18$, and the parameters of the ternary balanced block design are equal to $b_{3}=9 z, r_{3}=7 z, \quad k_{3}=7, \lambda_{3}=\rho_{13}=5 z, \rho_{23}=z, z=1,2, \ldots$ or $b_{3}=18, r_{3}=20, k_{3}=10, \lambda_{3}=22, \rho_{13}=16, \rho_{23}=2$, then $\mathbf{X} \in \mathbf{\Phi}_{n \times p}(-1,0,1)$ in the form (1) with the variance matrix of errors $\sigma^{2} \mathbf{I}_{n}$ is a regular D-optimal chemical balance weighing design.

Corollary 3.17. Let $v=9$. If the parameters of the balanced incomplete block design are equal to $b_{1}=12 t, r_{1}=4 t, k_{1}=3, \lambda_{1}=t, t=1,2, \ldots$, and the parameters of the balanced bipartite weighing design are equal to $b_{2}=18, r_{2}=10, k_{12}=1, k_{22}=4, \lambda_{12}=2, \lambda_{22}=3$ or $b_{2}=36, r_{2}=28, k_{12}=2, k_{22}=5, \lambda_{12}=10, \lambda_{22}=11$, and the parameters of the ternary balanced block design are equal to $b_{3}=r_{3}=z+8, k_{3}=9, \lambda_{3}=z+7, \rho_{13}=z, \rho_{23}=4, z=1,2, \ldots$ or $b_{3}=18, r_{3}=14, k_{3}=7, \lambda_{3}=9, \rho_{13}=2, \rho_{23}=6$, then $\mathbf{X} \in \mathbf{\Phi}_{n \times p}(-1,0,1)$ given in the form (1) with the variance matrix of errors $\sigma^{2} \mathbf{I}_{n}$ is a regular D-optimal chemical balance weighing design.

Corollary 3.18. Let $v=9$. The existence of the balanced incomplete block design with the parameters $b_{1}=12 t, r_{1}=4 t, k_{1}=3, \lambda_{1}=t, t=1,2, \ldots$, and the balanced bipartite weighing design with the parameters

$b_{2}=18, r_{2}=2(s+3), k_{12}=2, k_{22}=\lambda_{12}=s+1, \lambda_{22}=s, s=1,2$ or $b_{2}=36, r_{2}=12 s, k_{12}=s, k_{22}=2 s, \lambda_{12}=2 s^{2}, \lambda_{22}=\frac{s(5 s-3)}{2}, s=1,2$ and also the ternary balanced block design with the parameters $b_{3}=3(s+4)$, $r_{3}=2(s+4), \quad k_{3}=6, \lambda_{3}=s+5, \rho_{13}=8, \rho_{23}=s, s=1,2, \ldots, \quad$ implies the existence of the regular D-optimal chemical balance weighing design $\mathbf{X} \in \mathbf{\Phi}_{n \times p}(-1,0,1)$ in (1) with the variance matrix of errors $\sigma^{2} \mathbf{I}_{n}$. 
Corollary 3.19. Let $v=9$. The existence of the balanced incomplete block design with the parameters $b_{1}=12 t, r_{1}=4 t, k_{1}=3, \lambda_{1}=t, t=1,2, \ldots$, and the balanced bipartite weighing design with the parameters $b_{2}=36$, $r_{2}=32, k_{12}=3, k_{22}=5, \lambda_{12}=15, \lambda_{22}=13$ and also the ternary balanced block design with the parameters $b_{3}=18, r_{3}=10, k_{3}=5, \lambda_{3}=4$, $\rho_{13}=2, \rho_{23}=4$ implies the existence of the regular D-optimal chemical balance weighing design $\mathbf{X} \in \boldsymbol{\Phi}_{n \times p}(-1,0,1)$ in (1) with the variance matrix of errors $\sigma^{2} \mathbf{I}_{n}$.

Corollary 3.20. Let $v=10$. The existence of the balanced incomplete block design with the parameters $b_{1}=15, r_{1}=6, k_{1}=4, \lambda_{1}=2$ and the balanced bipartite weighing design with the parameters

$$
\begin{aligned}
& b_{2}=r_{2}=15, k_{12}=4, k_{22}=6, \lambda_{12}=8, \lambda_{22}=7 \text { or } \\
& b_{2}=45, r_{2}=27, k_{12}=2, k_{22}=4, \lambda_{12}=8, \lambda_{22}=7
\end{aligned}
$$

and also the ternary balanced block design with the parameters

$$
\begin{aligned}
& b_{3}=10, r_{3}=k_{3}=5, \lambda_{3}=2, \rho_{13}=3, \rho_{23}=1 \text { or } \\
& b_{3}=20, r_{3}=12, k_{3}=\lambda_{3}=\rho_{13}=6, \rho_{23}=3 \text { or } \\
& b_{3}=25, r_{3}=15, \quad k_{3}=6, \lambda_{3}=7, \rho_{13}=3, \rho_{23}=6,
\end{aligned}
$$

implies the existence of the regular D-optimal chemical balance weighing design $\mathbf{X} \in \boldsymbol{\Phi}_{n \times p}(-1,0,1)$ in (1) with the variance matrix of errors $\sigma^{2} \mathbf{I}_{n}$.

Corollary 3.21. Let $v=10$. The existence of the balanced incomplete block design with the parameters $b_{1}=18, r_{1}=9, k_{1}=5, \lambda_{1}=4$ and the balanced bipartite weighing design with the parameters

$$
\begin{aligned}
& b_{2}=r_{2}=30, k_{12}=3, k_{22}=7, \lambda_{12}=14, \lambda_{22}=16 \text { or } \\
& b_{2}=90, r_{2}=63, k_{12}=2, k_{22}=5, \lambda_{12}=20, \lambda_{22}=22
\end{aligned}
$$

and also the ternary balanced block design with the parameters

$$
\begin{aligned}
& b_{3}=10, r_{3}=k_{3}=7, \lambda_{3}=4, \rho_{13}=1, \rho_{23}=3 \text { or } \\
& b_{3}=10, r_{3}=k_{3}=8, \lambda_{3}=\rho_{13}=6, \rho_{23}=1 \text { or } \\
& b_{3}=20, r_{3}=14, k_{3}=7, \lambda_{3}=8, \rho_{13}=2, \rho_{23}=6,
\end{aligned}
$$

implies the existence of the regular D-optimal chemical balance weighing design $\mathbf{X} \in \boldsymbol{\Phi}_{n \times p}(-1,0,1)$ in (1) with the variance matrix of errors $\sigma^{2} \mathbf{I}_{n}$. 
Corollary 3.22. Let $v=10$. The existence of the balanced incomplete block design with the parameters $b_{1}=30, r_{1}=9, k_{1}=3, \lambda_{1}=2$ and the balanced bipartite weighing design with the parameters $b_{2}=30, r_{2}=21, k_{12}=3$, $k_{22}=4, \lambda_{12}=8, \lambda_{22}=6$ and also the ternary balanced block design with the parameters

$b_{3}=10 s, r_{3}=7 s, k_{3}=7, \lambda_{3}=4 s, \rho_{13}=s, \rho_{23}=3 s$ or $b_{3}=10 s, r_{3}=8 s, k_{3}=8, \lambda_{3}=\rho_{13}=6 s, \rho_{23}=s, s=1,2, \ldots$

implies the existence of the regular D-optimal chemical balance weighing design $\mathbf{X} \in \mathbf{\Phi}_{n \times p}(-1,0,1)$ in (1) with the variance matrix of errors $\sigma^{2} \mathbf{I}_{n}$.

Corollary 3.23. Let $v=11$. If the parameters of the balanced incomplete block design are equal to $b_{1}=11, r_{1}=k_{1}=5, \lambda_{1}=2$ and the parameters of balanced bipartite weighing design are equal to

$$
\begin{aligned}
& b_{2}=55, r_{2}=20, k_{12}=1, k_{22}=\lambda_{12}=\lambda_{22}=3 \text { or } \\
& b_{2}=55, r_{2}=45, k_{12}=3, k_{22}=6, \lambda_{12}=\lambda_{22}=18,
\end{aligned}
$$

and the parameters of the ternary balanced block design are equal to $b_{3}=11$, $r_{3}=k_{3}=7, \lambda_{3}=4, \rho_{13}=5, \rho_{23}=1$, then $\mathbf{X} \in \boldsymbol{\Phi}_{n \times p}(-1,0,1)$ in the form (1) with the variance matrix of errors $\sigma^{2} \mathbf{I}_{n}$ is a regular D-optimal chemical balance weighing design.

Corollary 3.24. Let $v=11$. If the parameters of the balanced incomplete block design are equal to $b_{1}=11, r_{1}=k_{1}=5, \lambda_{1}=2$ and the parameters of the balanced bipartite weighing design are equal to $b_{2}=55, r_{2}=25, k_{12}=1$, $k_{22}=\lambda_{12}=4, \lambda_{22}=6$, and also the parameters of the ternary balanced block design are equal to

$b_{3}=r_{3}=z+10, k_{3}=11, \lambda_{3}=z+9, \rho_{13}=z, \rho_{23}=5, z=1,2, \ldots$ or $b_{3}=22, r_{3}=20, k_{3}=10, \lambda_{3}=17, \rho_{13}=10, \rho_{23}=5$,

then $\mathbf{X} \in \mathbf{\Phi}_{n \times p}(-1,0,1)$ in the form (1) with the variance matrix of errors $\sigma^{2} \mathbf{I}_{n}$ is a regular $\mathrm{D}$-optimal chemical balance weighing design. 
Corollary 3.25. Let $v=11$. If the parameters of the balanced incomplete block design are equal to $b_{1}=11, r_{1}=k_{1}=5, \lambda_{1}=2$ and the parameters of the balanced bipartite weighing design are equal to

$b_{2}=r_{2}=11, k_{12}=5, k_{22}=\lambda_{12}=6, \lambda_{22}=5$ or

$b_{2}=11, r_{2}=10, k_{12}=k_{22}=\lambda_{12}=5, \lambda_{22}=4$ or

$b_{2}=55, r_{2}=15 s, k_{12}=s, k_{22}=2 s, \lambda_{12}=2 s^{2}, \lambda_{22}=\frac{s(5 s-3)}{2}, s=1,2$ or

$b_{2}=r_{2}=55, k_{12}=4, k_{22}=7, \lambda_{12}=28, \lambda_{22}=27$, and

also the parameters of the ternary balanced block design are equal to $b_{3}=22$, $r_{3}=14, k_{3}=7, \lambda_{3}=8, \rho_{13}=10, \rho_{23}=2$, then $\mathbf{X} \in \boldsymbol{\Phi}_{n \times p}(-1,0,1)$ in the form (1) with the variance matrix of errors $\sigma^{2} \mathbf{I}_{n}$ is a regular D-optimal chemical balance weighing design.

Corollary 3.26. Let $v=12$. If the parameters of the balanced incomplete block design are equal to $b_{1}=33, r_{1}=11, k_{1}=4, \lambda_{1}=3$ and the parameters of the balanced bipartite weighing design are equal to

$b_{2}=r_{2}=11, k_{12}=k_{22}=\lambda_{12}=6, \lambda_{22}=5$ or

$b_{2}=22, r_{2}=11, k_{12}=k_{22}=\lambda_{12}=3, \lambda_{22}=2$ or for $s=1,2, \ldots$ or

$b_{2}=33 s, r_{2}=11(2 s-1), k_{12}=2, k_{22}=2 s, \lambda_{12}=2 s^{2}, \lambda_{22}=\frac{s(5 s-3)}{2}$,

and the parameters of the ternary balanced block design are equal to

$b_{3}=4 z, r_{3}=3 z, k_{3}=9, \lambda_{3}=2 z, \rho_{13}=\rho_{23}=z, z=3,4, \ldots$ or

$b_{3}=12, r_{3}=k_{3}=10, \lambda_{3}=\rho_{13}=8, \rho_{23}=1$,

then $\mathbf{X} \in \mathbf{\Phi}_{n \times p}(-1,0,1)$ in the form (1) with the variance matrix of errors $\sigma^{2} \mathbf{I}_{n}$ is a regular D-optimal chemical balance weighing design.

Corollary 3.27. Let $v=12$. If the parameters of the balanced incomplete block design are equal to $b_{1}=33, r_{1}=11, k_{1}=4, \lambda_{1}=3$ and the parameters of the balanced bipartite weighing design are equal to

$b_{2}=44, r_{2}=33, k_{12}=3, k_{22}=6, \lambda_{12}=\lambda_{22}=12$ or

$b_{2}=66, r_{2}=22, k_{12}=1, k_{22}=\lambda_{12}=\lambda_{22}=3$ or

$b_{2}=132, r_{2}=44, k_{12}=1, k_{22}=3, \lambda_{12}=\lambda_{22}=6$,

and the parameters of the ternary balanced block design are equal to $b_{3}=18$, $r_{3}=15, k_{3}=10, \lambda_{3}=11, \rho_{13}=1, \rho_{23}=7$, then $\mathbf{X} \in \boldsymbol{\Phi}_{n \times p}(-1,0,1)$ in the 
form (1) with the variance matrix of errors $\sigma^{2} \mathbf{I}_{n}$ is a regular D-optimal chemical balance weighing design.

Corollary 3.28. Let $v=13$. The existence of the balanced incomplete block design with the parameters $b_{1}=13, r_{1}=k_{1}=4, \lambda_{1}=1$ and the balanced bipartite weighing design with the parameters

$b_{2}=13, r_{2}=12, k_{12}=k_{22}=\lambda_{12}=6, \lambda_{22}=5$ or

$b_{2}=26, r_{2}=12, k_{12}=k_{22}=\lambda_{12}=3, \lambda_{22}=2$ or

$b_{2}=26, r_{2}=14, k_{12}=3, k_{22}=\lambda_{12}=4, \lambda_{22}=3$ or

$b_{2}=26, r_{2}=20, k_{12}=4, k_{22}=6, \lambda_{12}=8, \lambda_{22}=7$ or

$b_{2}=r_{2}=39, k_{12}=5, k_{22}=8, \lambda_{12}=20, \lambda_{22}=19$ or

$b_{2}=78, r_{2}=18 s, k_{12}=s, k_{22}=2 s, \lambda_{12}=2 s^{2}, \lambda_{22}=\frac{s(5 s-3)}{2}, s=1,2$ or

$b_{2}=78, r_{2}=66, k_{12}=4, k_{22}=7, \lambda_{12}=28, \lambda_{22}=27$,

and also the ternary balanced block design with the parameters

$b_{3}=13, r_{3}=k_{3}=10, \lambda_{3}=7, \rho_{13}=4, \rho_{23}=3$ or

$b_{3}=13, r_{3}=k_{3}=11, \lambda_{3}=\rho_{13}=9, \rho_{23}=1$,

implies the existence of the regular D-optimal chemical balance weighing design $\mathbf{X} \in \mathbf{\Phi}_{n \times p}(-1,0,1)$ in (1) with the variance matrix of errors $\sigma^{2} \mathbf{I}_{n}$.

Corollary 3.29. Let $v=13$. The existence of the balanced incomplete block design with the parameters $b_{1}=13, r_{1}=4, k_{1}=4, \lambda_{1}=1$ and the balanced bipartite weighing design with the parameters

$b_{2}=13 s, r_{2}=9 s, k_{12}=3, k_{22}=6, \lambda_{12}=\lambda_{22}=3 s, s=1,2,3,4,6$ or

$b_{2}=26 s, r_{2}=8 s, k_{12}=1, k_{22}=3, \lambda_{12}=\lambda_{22}=s, s=1,2$ and

also the ternary balanced block design with the parameters $b_{3}=r_{3}=z+12$, $k_{3}=13, \lambda_{3}=z+11, \rho_{13}=z, \rho_{23}=6, z=1,2, \ldots$, implies the existence of the regular D-optimal chemical balance weighing design $\mathbf{X} \in \boldsymbol{\Phi}_{n \times p}(-1,0,1)$ in (1) with the variance matrix of errors $\sigma^{2} \mathbf{I}_{n}$.

Corollary 3.30. Let $v=13$. The existence of the balanced incomplete block design with the parameters $b_{1}=26, r_{1}=8, k_{1}=4, \lambda_{1}=2$ and the balanced bipartite weighing design with the parameters 
$b_{2}=13, r_{2}=12, k_{12}=k_{22}=\lambda_{12}=6, \lambda_{22}=5$ or

$b_{2}=26, r_{2}=12, k_{12}=k_{22}=\lambda_{12}=3, \lambda_{22}=2$ or

$b_{2}=26, r_{2}=14, k_{12}=3, k_{22}=\lambda_{12}=4, \lambda_{22}=3$ or

$b_{2}=26, r_{2}=20, k_{12}=4, k_{22}=6, \lambda_{12}=8, \lambda_{22}=7$ or

$b_{2}=39, r_{2}=3 s, k_{12}=2, k_{22}=\lambda_{12}=s-2, \lambda_{22}=s-3, s=4,5$ or

$b_{2}=r_{2}=39, k_{12}=5, k_{22}=8, \lambda_{12}=20, \lambda_{22}=19$ or

$b_{2}=78, r_{2}=18 s, k_{12}=s, k_{22}=2 s, \lambda_{12}=2 s^{2}, \lambda_{22}=\frac{s(5 s-3)}{2}, s=1,2$ or

$b_{2}=78, r_{2}=66, k_{12}=4, k_{22}=7, \lambda_{12}=28, \lambda_{22}=27$ and

also the ternary balanced block design with the parameters $b_{3}=r_{3}=z+12$, $k_{3}=13, \lambda_{3}=z+11, \rho_{13}=z, \rho_{23}=6, z=1,2,3$, implies the existence of the regular D-optimal chemical balance weighing design $\mathbf{X} \in \boldsymbol{\Phi}_{n \times p}(-1,0,1)$ in (1) with the variance matrix of errors $\sigma^{2} \mathbf{I}_{n}$.

Corollary 3.31. Let $v=15$. The existence of the balanced incomplete block design with the parameters $b_{1}=15, r_{1}=k_{1}=7, \lambda_{1}=3$ and the balanced bipartite weighing design with the parameters $b_{2}=105, r_{2}=49, k_{12}=2$, $k_{22}=5, \lambda_{12}=10, \lambda_{22}=11$, and also the ternary balanced block design with the parameters

$b_{3}=15, r_{3}=k_{3}=12, \lambda_{3}=9, \rho_{13}=6, \rho_{23}=3$ or

$b_{3}=15, r_{3}=k_{3}=13, \lambda_{3}=\rho_{13}=11, \rho_{23}=1$,

implies the existence of the regular D-optimal chemical balance weighing design $\mathbf{X} \in \mathbf{\Phi}_{n \times p}(-1,0,1)$ in (1) with the variance matrix of errors $\sigma^{2} \mathbf{I}_{n}$.

Corollary 3.32. Let $v=15$. If the parameters of the balanced incomplete block design are equal to $b_{1}=15, r_{1}=k_{1}=7, \lambda_{1}=3$ and the parameters of the balanced bipartite weighing design are equal to

$b_{2}=r_{2}=15, k_{12}=7, k_{22}=\lambda_{12}=8, \lambda_{22}=7$ or

$b_{2}=105, r_{2}=21 s, k_{12}=s, k_{22}=2 s, \lambda_{12}=2 s^{2}, \lambda_{22}=\frac{s(5 s-3)}{2}, s=1,2$,

and the parameters of the ternary balanced block design are equal to $b_{3}=15$, $r_{3}=k_{3}=9, \lambda_{3}=5, \rho_{13}=7, \rho_{23}=1$, then $\mathbf{X} \in \boldsymbol{\Phi}_{n \times p}(-1,0,1)$ in the form (1) with the variance matrix of errors $\sigma^{2} \mathbf{I}_{n}$ is a regular D-optimal chemical balance weighing design. 
Corollary 3.33. Let $v=15$. The existence of the balanced incomplete block design with the parameters $b_{1}=42, r_{1}=14, k_{1}=5, \lambda_{1}=4$ and the balanced bipartite weighing design with the parameters $b_{2}=35 s, r_{2}=21 s$, $k_{12}=3, k_{22}=6, \lambda_{12}=\lambda_{22}=6 s, s=1,2, \ldots$, and also the ternary balanced block design with the parameters $b_{3}=r_{3}=30, k_{3}=15, \lambda_{3}=28, \rho_{13}=2$, $\rho_{23}=14$, implies the existence of the regular D-optimal chemical balance weighing design $\mathbf{X} \in \mathbf{\Phi}_{n \times p}(-1,0,1)$ in (1) with the variance matrix of errors $\sigma^{2} \mathbf{I}_{n}$

Corollary 3.34. Let $v=16$. The existence of the balanced incomplete block design with the parameters

$b_{1}=8 t, r_{1}=5 t, k_{1}=10, \lambda_{1}=3 t, t=2,3, \ldots$ or

$b_{1}=8 t, r_{1}=3 t, k_{1}=6, \lambda_{1}=t, t=2,3 \ldots$,

and the balanced bipartite weighing design with the parameters

$b_{2}=40 s, r_{2}=10 s, k_{12}=1, k_{22}=3, \lambda_{12}=\lambda_{22}=s, s=1,3$ or

$b_{2}=80, r_{2}=45, k_{12}=3, k_{22}=6, \lambda_{12}=\lambda_{22}=12$,

and also the ternary balanced block design with the parameters

$b_{3}=16, r_{3}=k_{3}=13, \lambda_{3}=10, \rho_{13}=7, \rho_{23}=3$ or

$b_{3}=16, r_{3}=k_{3}=14, \lambda_{3}=12, \rho_{13}=12, \rho_{23}=1$ or

$b_{3}=20, r_{3}=14, k_{3}=8, \lambda_{3}=\rho_{13}=6, \rho_{23}=4$,

implies the existence of the regular D-optimal chemical balance weighing design $\mathbf{X} \in \mathbf{\Phi}_{n \times p}(-1,0,1)$ in (1) with the variance matrix of errors $\sigma^{2} \mathbf{I}_{n}$.

Corollary 3.35. Let $v=17$. The existence of the balanced incomplete block design with the parameters $b_{1}=34, r_{1}=16, k_{1}=8, \lambda_{1}=7$ and the balanced bipartite weighing design with the parameters

$b_{2}=68, r_{2}=20, k_{12}=1, k_{22}=4, \lambda_{12}=2, \lambda_{22}=3$ or

$b_{2}=136, r_{2}=56, k_{12}=2, k_{22}=5, \lambda_{12}=10, \lambda_{22}=11$,

and also the ternary balanced block design with the parameters $b_{3}=17, r_{3}=$ $k_{3}=12, \lambda_{3}=8, \rho_{13}=8, \rho_{23}=2$, implies the existence of the regular 
D-optimal chemical balance weighing design $\mathbf{X} \in \mathbf{\Phi}_{n \times p}(-1,0,1)$ in (1) with the variance matrix of errors $\sigma^{2} \mathbf{I}_{n}$.

Corollary 3.36. Let $v=18$. The existence of the balanced incomplete block design with the parameters

$b_{1}=34, r_{1}=17, k_{1}=9, \lambda_{1}=8$ or

$b_{1}=306, r_{1}=119, k_{1}=7, \lambda_{1}=42$ and

the balanced bipartite weighing design with the parameters $b_{2}=34, r_{2}=17$, $k_{12}=3, k_{22}=6, \lambda_{12}=\lambda_{22}=4$ and also the ternary balanced block design with the parameters $b_{3}=18, r_{3}=k_{3}=11, \lambda_{3}=6, \rho_{13}=3, \rho_{23}=4$ implies the existence of the regular D-optimal chemical balance weighing design $\mathbf{X} \in \boldsymbol{\Phi}_{n \times p}(-1,0,1)$ in (1) with the variance matrix of errors $\sigma^{2} \mathbf{I}_{n}$.

Proof: The proof is analogous to that of Theorem 3.3.

Corollary 3.37. Let $v=19$. If the parameters of the balanced incomplete block design are equal to $b_{1}=19, r_{1}=k_{1}=9, \lambda_{1}=4$ and the parameters of the balanced bipartite weighing design are equal to $b_{2}=171, r_{2}=63, k_{12}=2$, $k_{22}=5, \lambda_{12}=10, \lambda_{22}=11$, and the parameters of the ternary balanced block design are equal to $b_{3}=19, r_{3}=k_{3}=15, \lambda_{3}=11, \rho_{13}=3, \rho_{23}=6$ or $b_{3}=19, r_{3}=k_{3}=17, \lambda_{3}=\rho_{13}=15, \rho_{23}=1$, then $\mathbf{X} \in \boldsymbol{\Phi}_{n \times p}(-1,0,1)$ in the form (1) with the variance matrix of errors $\sigma^{2} \mathbf{I}_{n}$ is a regular D-optimal chemical balance weighing design.

Corollary 3.38. Let $v=19$. The existence of the balanced incomplete block design with the parameters $b_{1}=19, r_{1}=k_{1}=9, \lambda_{1}=4$ and the balanced bipartite weighing design with the parameters

$b_{2}=19 s, r_{2}=9 s, k_{12}=3, k_{22}=6, \lambda_{12}=\lambda_{22}=2 s, s=1,2,3,6,9$ or

$b_{2}=57 s, r_{2}=12 s, k_{12}=1, k_{22}=3, \lambda_{12}=\lambda_{22}=s, s=1,3$,

and also the ternary balanced block design with the parameters

$b_{3}=19, r_{3}=k_{3}=13, \lambda_{3}=8, \rho_{13}=1, \rho_{23}=6$ or

$b_{3}=19, r_{3}=k_{3}=14, \lambda_{3}=10, \rho_{13}=12, \rho_{23}=1$, 
implies the existence of the regular D-optimal chemical balance weighing design $\mathbf{X} \in \boldsymbol{\Phi}_{n \times p}(-1,0,1)$ in (1) with the variance matrix of errors $\sigma^{2} \mathbf{I}_{n}$.

Corollary 3.39. Let $v=19$. If the parameters of the balanced incomplete block design are equal to $b_{1}=19, r_{1}=k_{1}=9, \lambda_{1}=4$ and the parameters of the balanced bipartite weighing design are equal to $b_{2}=171, r_{2}=45, k_{12}=1$, $k_{22}=\lambda_{12}=4, \lambda_{22}=6$, and also parameters of the ternary balanced block design are equal to $b_{3}=r_{3}=k_{3}=19, \lambda_{3}=18, \rho_{13}=1, \rho_{23}=9$, then $\mathbf{X} \in \boldsymbol{\Phi}_{n \times p}(-1,0,1)$ in the form (1) with the variance matrix of errors $\sigma^{2} \mathbf{I}_{n}$ is a regular D-optimal chemical balance weighing design.

Corollary 3.40. Let $v=19$. If the parameters of the balanced incomplete block design are equal to $b_{1}=19, r_{1}=k_{1}=9, \lambda_{1}=4$ and the parameters of the balanced bipartite weighing design are equal to $b_{2}=171, r_{2}=54, k_{12}=2$, $k_{22}=4, \lambda_{12}=8, \lambda_{22}=7, \quad$ or $\quad b_{2}=19 s, r_{2}=s+18, k_{12}=12-3 s$, $k_{22}=\lambda_{12}=13-3 s, \lambda_{22}=12-3 s, s=1,3$, and also the parameters of the ternary balanced block design are equal to $b_{3}=19, r_{3}=k_{3}=12, \lambda_{3}=7$, $\rho_{13}=6, \rho_{23}=3$, then $\mathbf{X} \in \boldsymbol{\Phi}_{n \times p}(-1,0,1)$ in the form (1) with the variance matrix of errors $\sigma^{2} \mathbf{I}_{n}$ is a regular D-optimal chemical balance weighing design.

Corollary 3.41. Let $v=4 s+1$. The existence of the balanced incomplete block design with the parameters $b_{1}=2(4 s+1), r_{1}=4 s, k_{1}=2 s, \lambda_{1}=2 s-1$, the balanced bipartite weighing design with the parameters $b_{2}=s(4 s+1)$, $r_{2}=8 s, k_{12}=2, k_{22}=6, \lambda_{12}=6, \lambda_{22}=8$, and the ternary balanced block design with the parameters:

$b_{3}=u(4 s+1), r_{3}=u(4 s-1), k_{3}=4 s-1, \lambda_{3}=u(4 s-3), \rho_{13}=u(4 s-3)$,

$\rho_{23}=u, u=1,2, \ldots, s=2,3, \ldots$, except for the case $u=1, s=1$ or

$b_{3}=u(4 s+1), r_{3}=u(4 s-2), k_{3}=4 s-2, \lambda_{3}=u(4 s-5), \rho_{13}=$ $u(4 s-8), \rho_{23}=3 u, u=1,2, \ldots, s=3,4, \ldots$ or $b_{3}=u(4 s+1), r_{3}=u(4 s-3), \quad k_{3}=4 s-3, \lambda_{3}=u(4 s-7), \rho_{13}=$ $u(4 s-15), \rho_{23}=6 u, u=1,2, \ldots, s=3,4, \ldots$, implies the existence of the 
regular D-optimal chemical balance weighing design $\mathbf{X} \in \mathbf{\Phi}_{n \times p}(-1,0,1)$ in (1) with the variance matrix of errors $\sigma^{2} \mathbf{I}_{n}$.

Corollary 3.42. Let $v=(2 s+1)^{2}$. The existence of the balanced incomplete block design with the parameters $b_{1}=4(2 s+1) t, r_{1}=4 s t, k_{1}=s(2 s+1)$, $\lambda_{1}=(2 s-1) t, 4 t \geq 2 s+1, s=2,3,5,6,8,9$, the balanced bipartite weighing design with the parameters $b_{2}=2 s(s+1)(2 s+1)^{2} u / 3, r_{2}=8 s u(s+1) / 3$, $k_{12}=1, k_{22}=3, \lambda_{12}=\lambda_{22}=u, u \equiv 0 \bmod 3$, and the ternary balanced block design with the parameters

$b_{3}=(2 s+1)^{2} w, r_{3}=\left(4 s^{2}+4 s-1\right) w, k_{3}=4 s^{2}+4 s-1, \lambda_{3}=\left(4 s^{2}+\right.$ $4 s-3) w, \rho_{13}=\left(4 s^{2}+4 s-3\right) w, \rho_{23}=w, w=1,2, \ldots$ or

$b_{3}=(2 s+1)^{2} w, r_{3}=\left(4 s^{2}+4 s-2\right) w, k_{3}=4 s^{2}+4 s-2, \lambda_{3}=\left(4 s^{2}+\right.$ $4 s-5) w, \rho_{13}=\left(4 s^{2}+4 s-8\right) w, \rho_{23}=3 w, w=1,2, \ldots$ or

$b_{3}=(2 s+1)^{2} w, \quad r_{3}=\left(4 s^{2}+4 s-3\right) w, k_{3}=4 s^{2}+4 s-3, \lambda_{3}=\left(4 s^{2}+\right.$ $4 s-7) w, \rho_{13}=\left(4 s^{2}+4 s-15\right) w, \rho_{23}=6 w, w=1,2, \ldots$,

implies the existence of the regular D-optimal chemical balance weighing design $\mathbf{X} \in \boldsymbol{\Phi}_{n \times p}(-1,0,1)$ in (1) with the variance matrix of errors $\sigma^{2} \mathbf{I}_{n}$.

\section{Examples}

Let us consider an experiment in which we determine unknown measurements of $p=5$ objects and $n=30$ measurements. According to Theorem 3.3 we consider the balanced incomplete block design with the parameters $v=5, b_{1}=10$, $r_{1}=4, k_{1}=2, \lambda_{1}=1$ and the incidence matrix $\mathbf{N}_{1}$, the balanced bipartite weighing design with the parameters $v=5, b_{2}=r_{2}=5, k_{12}=1, k_{22}=4$, $\lambda_{12}=2, \lambda_{22}=3$ and the incidence matrix $\mathbf{N}_{2}^{*}$, and also the ternary balanced block design with the parameters $v=5 b_{3}=15, r_{3}=9, k_{3}=3, \lambda_{3}=4$, $\rho_{13}=7, \rho_{23}=1$ and the incidence matrix $\mathbf{N}_{3}$, where 


$$
\begin{gathered}
\boldsymbol{N}_{1}=\left[\begin{array}{llllllllll}
1 & 1 & 0 & 0 & 0 & 0 & 1 & 0 & 1 & 0 \\
1 & 0 & 1 & 0 & 1 & 0 & 0 & 0 & 0 & 1 \\
0 & 1 & 1 & 0 & 0 & 1 & 0 & 1 & 0 & 0 \\
0 & 0 & 0 & 1 & 0 & 0 & 0 & 1 & 1 & 1 \\
0 & 0 & 0 & 1 & 1 & 1 & 1 & 0 & 0 & 0
\end{array}\right], \boldsymbol{N}_{2}^{*}=\left[\begin{array}{lllllll}
1_{1} & 1_{2} & 1_{2} & 1_{2} & 1_{2} \\
1_{2} & 1_{1} & 1_{2} & 1_{2} & 1_{2} \\
1_{2} & 1_{2} & 1_{1} & 1_{2} & 1_{2} \\
1_{2} & 1_{2} & 1_{2} & 1_{1} & 1_{2} \\
1_{2} & 1_{2} & 1_{2} & 1_{2} & 1_{1}
\end{array}\right] \\
\boldsymbol{N}_{3}=\left[\begin{array}{lllllllllllllll}
2 & 1 & 1 & 0 & 1 & 0 & 1 & 0 & 0 & 1 & 0 & 1 & 1 & 0 & 0 \\
1 & 1 & 1 & 1 & 0 & 1 & 0 & 2 & 1 & 0 & 1 & 0 & 0 & 0 & 0 \\
0 & 0 & 1 & 0 & 1 & 0 & 1 & 1 & 1 & 1 & 0 & 0 & 0 & 2 & 1 \\
0 & 1 & 0 & 0 & 0 & 1 & 0 & 0 & 1 & 0 & 1 & 1 & 2 & 1 & 1 \\
0 & 0 & 0 & 2 & 1 & 1 & 1 & 0 & 0 & 1 & 1 & 1 & 1 & 0 & 1
\end{array}\right]
\end{gathered}
$$

Here, $1_{h}$ denotes the element belonging to the $h^{\text {th }}$ subblock, $h=1,2$. Thus the design matrix of the regular D-optimal chemical balance weighing design $\mathbf{X} \in \boldsymbol{\Phi}_{30 \times 5}(-1,0,1)$ is given in the form $\mathbf{X}^{\prime}=\left[\begin{array}{lll}\boldsymbol{X}_{\mathbf{1}}^{\prime} & \boldsymbol{X}_{\mathbf{2}}^{\prime} & \boldsymbol{X}_{3}^{\prime}\end{array}\right]$, where

$$
\boldsymbol{X}_{\mathbf{1}}=\left[\begin{array}{rrrrr}
1 & 1 & -1 & -1 & -1 \\
1 & -1 & 1 & -1 & -1 \\
-1 & 1 & 1 & -1 & -1 \\
-1 & -1 & -1 & 1 & 1 \\
-1 & 1 & -1 & -1 & 1 \\
-1 & -1 & 1 & -1 & 1 \\
1 & -1 & -1 & -1 & 1 \\
-1 & -1 & 1 & 1 & -1 \\
1 & -1 & -1 & 1 & -1 \\
-1 & 1 & -1 & 1 & -1
\end{array}\right], \boldsymbol{X}_{\mathbf{3}}=\left[\begin{array}{rrrrr}
1 & 0 & -1 & -1 & -1 \\
0 & 0 & -1 & 0 & -1 \\
0 & 0 & 0 & -1 & -1 \\
-1 & 0 & -1 & -1 & 1 \\
0 & -1 & 0 & -1 & 0 \\
-1 & 0 & -1 & 0 & 0 \\
0 & -1 & 0 & -1 & 0 \\
-1 & 1 & 0 & -1 & -1 \\
-1 & 0 & 0 & 0 & -1 \\
0 & -1 & 0 & -1 & 0 \\
-1 & 0 & -1 & 0 & 0 \\
0 & -1 & -1 & 0 & 0 \\
0 & -1 & -1 & 1 & -1 \\
-1 & -1 & 1 & 0 & -1 \\
-1 & -1 & 0 & 0 & 0
\end{array}\right]
$$

and $\boldsymbol{X}_{\mathbf{2}}=\mathbf{1}_{\mathbf{5}} \mathbf{1}_{\mathbf{5}}^{\prime}-2 \mathbf{I}_{\mathbf{5}}$.

\section{Case study}

Some constructions of the design matrices of regular D-optimal chemical balance weighing designs are presented in the literature. They are based on different incidence matrices of known block designs. However, it is not possible to construct an optimal design matrix for any number of objects and measurements. Hence, the main significance of Corollaries 3.2-3.42 is that they make it possible to broaden the classes in which regular D-optimal chemical balance weighing 
designs exist, as given in the literature: see for example Ceranka and Graczyk (2018). In this paper, we discuss regular D-optimal weighing designs where the errors are uncorrelated and have the same variance. In Ceranka and Graczyk (2018) a regular D-optimal weighing design with nonnegative correlations of errors is considered. Under the assumption $\rho=0$, based on a result given in Ceranka and Graczyk (2018), we can determine a regular D-optimal chemical balance weighing design for $p=7$ objects and 42 measurements. In this paper, we are able to establish a regular D-optimal chemical balance weighing design in the class $\boldsymbol{\Phi}_{35 \times 7}(-1,0,1)$ (Corollary 3.7). Moreover, based on Ceranka and Graczyk (2018) we can determine a regular D-optimal chemical balance weighing design in the class $\boldsymbol{\Phi}_{136 \times 16}(-1,0,1)$, and based on Corollary 3.31 above we can do so in the class $\boldsymbol{\Phi}_{72 \times 16}(-1,0,1)$. On the other hand, based on the method given in Ceranka and Graczyk (2018), a regular D-optimal chemical balance weighing design is determined in the class $\boldsymbol{\Phi}_{22 \times 11}(-1,0,1)$, while based on the method presented here the same is done in the class $\boldsymbol{\Phi}_{44 \times 11}(-1,0,1)$. In fact, the problem of determining a D-optimal chemical balance weighing design in any class $\mathbf{X} \in \mathbf{\Phi}_{n \times p}(-1,0,1)$ is still open and requires further study.

\section{Discussion}

Nowadays, such designs have applications in many different branches of knowledge, such as medicine, agriculture and industry; see Jacroux (2009), as well as the economic survey given in Ceranka and Graczyk (2014). Applications can also be found, among others, in bioenergetics.

The presented D-optimal chemical balance weighing design may be applicable in determining the thermal expansion of plutonium oxide pellets. This problem is considered in the literature (Beckman, 1973). Pellet expansion can be measured using a dilatometer, where the pellet is surrounded on three sides by aluminum. When the pellet expands with increasing temperature, the aluminum casing also expands. Therefore, the difference between the thermal expansion of the plutonium pellet and of the aluminum casing is the expansion measured. To 
estimate the plutonium's thermal expansion the design presented above can be used, where vectors of unknown parameters for platinum, aluminum and plutonium can be considered.

Cheng (1980) applied results on chemical weighing designs to the setting of $2^{n}$ fractional designs. We relate the coded levels of factors $(-1,0,+1)$ in the plan of factorial designs to $(-1,0,1)$ in the experimental plan of chemical balance weighing designs which is considered above. These designs have been used for to the study of the effect of drying conditions on $\alpha$-tocopherol content in prickly pear seed oil (Morti et al., 2015). The optimization of the variables affecting the quality of the oil was carried out following a $2^{3}$ complete factorial design. The measured response was the amount of tocopherol in the oil extracted from prickly pear seeds. Three factors were studied: temperature, relative humidity, and air velocity for convective drying.

In the present paper, we deal with factors having three levels $(-1,0,1)$, namely experiment type $3^{\tau}$. By the use of a three-level fractional design, the effects of a large number of factors can be studied. Having three levels allows for some assessment of curvature in the factor-response relationship. This type of experiment is presented in Jones and Nachtsheim (2011). In the future, consideration may be given to the possibility of determining experimental plans based on D-optimal matrices for other experiments where the factors have three levels.

\section{REFERENCES}

Beckman R.J. (1973). An application of multivariate weighing designs. Communication in Statistics 1(6), 561-565.

Billington E.J. (1984). Balanced n-array designs: a combinatorial survey and some new results. Ars Combinatoria 17A, 37-72.

Cheng C.S. (1980). Optimality of some weighing and $2^{\mathrm{n}}$ fractional factorial designs. Annals of Statistics 8, 436-446.

Ceranka B., Graczyk M. (2004a). Balanced ternary block designs under the certain condition. Colloquium Biometricum 34, 63-75.

Ceranka B., Graczyk M. (2004b). Balanced bipartite weighing designs under the certain condition. Colloquium Biometricum 34a, 17-28. 
Ceranka B., Graczyk M. (2016). New construction of D-optimal weighing design with non-negative correlations of errors. Colloquium Biometricum 46, 31-45.

Ceranka B., Graczyk M. (2017). Some D-optimal chemical balance weighing designs: theory and examples. Biometrical Letters 54(2), 137-154.

Ceranka B., Graczyk M. (2018). Regular D-optimal weighing design with non-negative correlations of errors constructed from some block designs. Colloquium Biometricum 48, 1-17.

Huang, C. (1976). Balanced bipartite block design. Journal of Combinatorial Theory (A), $21,20-34$.

Jacroux M., Wong C.S., Masaro J.C. (1983). On the optimality of chemical balance weighing design. Journal of Statistical Planning and Inference 8, 213-240.

Katulska K., Smaga Ł. (2013). A note on D-optimal chemical balance weighing designs and their applications. Colloquium Biometricum 43, 37-45.

Motri S., Touil A., Benselma Z., Hassini L., Bettaieb E., Zagrouba F. (2015). Application of factorial design to the study of the effect of drying conditions on $\alpha$-tocopherol content in prickly pear seed oil. Journal of New Sciences 19(8), 759-765.

Raghavarao, D. (1971). Constructions and Combinatorial Problems in designs of Experiments. John Wiley Inc., New York.

Sathe Y.S., Shenoy R.G. (1990). Construction method for some A- and D-optimal weighing designs when $\mathrm{N} \equiv 3(\bmod 4)$. Journal of Statistical Planning and Inference $24,369-375$. 